\title{
International Conference cum workshop on Analysis and its Applications June 18-22, 2018 Indian Institute of Technology Madras, Chennai, India
}

\author{
G. Ramesh ${ }^{1}$ - T. S. S. R. K. Rao ${ }^{2}$ K. C. Sivakumar ${ }^{3}$
}

Received: 30 April 2021 / Accepted: 8 May 2021 / Published online: 29 June 2021

(C) Forum D'Analystes, Chennai 2021

A Conference-cum-workshop on Analysis and its Applications was held at the Indian Institute of Technology Madras, during June 18-22, 2018. One of the primary objectives of that event was to commemorate the 65th birthday of Professor S.H. Kulkarni, fondly referred to as SHK. This provided a platform for many of SHK's students and collaborators to share both their professional and personal experiences that they had with SHK. The conference was made possible through funding from the SERB, Government of India and NBHM, DAE, Government of India. Additional funding also was received from the Office of Alumni and International Relations, the department of Mathematics, Indian Institute of Technology Madras (IIT Madras).

In what follows, we provide a brief review of contributions to this special issue, a short biography of Professor SHK, and a list of his publications. The Guest Editors would like to thank all the authors, the Editors-in-Chief of the Journal of Analysis, and all the referees who provided peer review. This special issue would not have been possible without their valuable time and inputs. We regret the inordinate delay in bringing out this issue due to circumstances beyond our control (pandemic included).

K. C. Sivakumar

kcskumar@iitm.ac.in

G. Ramesh

rameshg@math.iith.ac.in

T. S. S. R. K. Rao

tssrkrao@gmail.com

1 Department of Mathematics, Indian Institute of Technology, Hyderabad, Telangana, India

2 Ashoka University, Rai, Haryana, India

3 Department of Mathematics, Indian Institute of Technology Madras, Chennai, India 


\section{A brief overview of the contents}

In their survey article, "A short survey on open problems in metric fixed point theory and some related results for nonexpansive mappings", Dutta and Veeramani present some open problems in metric fixed point theory and discuss a few related results for nonexpansive mappings.

John, Kulkarni and Radha, in their article "Shift Invariant spaces in $L^{2}\left(\mathbb{R}, \mathbb{C}^{m}\right)$ with $m$ generators" analyze the regular sampling and reconstruction problem over certain classes of shift invariant subspaces of $L^{2}\left(\mathbb{R}, \mathbb{C}^{m}\right)$. The authors consider certain signal spaces, where it is shown that most of the existing results have appropriate analogues.

Kalauch, Lavanya and Sivakumar, continuing their earlier work on singular M-operators, show how some interesting results on singular irreducible M-matrices have analogues to irreducible operators over ordered Banach spaces.

The Korovkin-type theorems obtained in the context of matrix/operator sequences unify several preconditioning techniques such as Fourier, Hartley etc. These results find applications in spectral approximation problems for bounded selfadjoint operators. Kiran Kumar, Namboodiri and Rahul Rajan, in their work " $A$ short survey on preconditioners and Korovkin-type theorems", present a short survey of all these developments.

Kühner studies the problem of characterizing the long term behaviour of a semiflow on a compact space employing the asymptotic properties of the corresponding Koopman semigroup. Specifically, in Kühner's article titled "What can Koopmanism do for attractors in dynamical systems?", she presents comparisons between different concepts of attractors, such as asymptotically stable attractors, Milnor attractors and centers of attraction. Moreover, she presents a characterization for the minimal attractor for each of the property mentioned here. Since the Koopman semigroup allows a global linearization for a possibly nonlinear semiflow, as the author observes, the vital aspect is that one only needs techniques and results for linear operator semigroups.

Kulkarni and Ramesh propose and study a new class of unbounded operators which attain minimum modulus. A relationship between this class and the class of norm attaining bounded operators is obtained and comparisons between these two classes are presented. They also define absolutely minimum attaining operators (that are not necessarily bounded) and characterize injective absolutely minimum attaining operators as those with compact generalized inverse. Among other things, it is shown that every such operator has a non trivial hyperinvariant subspace.

A nice survey article by Kulkarni on "Spectrum and related results, A survey" deals with variations on the spectrum of an element in a Banach algebra. The notions covered include, $\epsilon$-conditional spectrum, $\epsilon$-pseudo spectrum and $(n, \epsilon)$ pseudo spectrum. This article covers the work done during the past decade by the author and his collaborators.

In his survey article, "Regularization of Ill-Posed Operator Equations: An Overview”, Nair discusses stability issues associated with ill-posed linear operator 
equations and the need to have regularization methods for obtaining stable approximate solutions. By way of illustration, he describes the Lavrentiev regularization in the setting of Banach spaces and Tikhonov regularization in the setting of Hilbert spaces, and presents error estimates in each of the cases. Procedures to obtain error estimates under milder general source conditions together with better error estimates are also outlined.

Prasad derives several generalizations of the well known core-nilpotent decomposition of a square matrix and studies their properties, in his article "Generalized CoreNilpotent Decomposition”. He shows how the core-EP decomposition and EP-nilpotent decompositions may be obtained as special cases.

Let $X$ denote a complex Banach space and $L(X)$, the Banach space of all bounded linear operators on $X$. Let $\Omega \subset \mathbb{C}$ be open and connected. Let $T, V: \Omega \rightarrow L(X)$ be holomorphic operator-valued functions. In the article "Perturbations of discrete spectra of holomorphic operator-valued functions" Rafikul Alam and Jibrail Ali, consider the one parameter family of operator-valued functions

$$
W(\lambda, t):=T(\lambda)+t V(\lambda), t \in \mathbb{C},
$$

and analyze evolution of the discrete eigenvalues of $W(\lambda, t)$ when $t \in \mathbb{C}$. A brief review of the discrete spectrum of $T(\lambda)$ and several equivalent characterizations for discrete eigenvalues of $T(\lambda)$ are presented.

In the article "Boundaries for the Cartesian product of real function algebras," Roghelia, Mehta and Mehta study the notions of Choquet sets and Silov boundary for the Cartesian product of real function algebras and function spaces and obtain some interesting results.

"Equiangular lines in $\mathbb{R}^{d}$ " is the title of the short survey by Sane, where he considers a curious interplay between a graph theoretic problem and linear algebra. The linear algebraic problem is to maximize the number of lines through the origin that make the same angle with each other, given the dimension $d$ of the real space $\mathbb{R}^{d}$.

Sofi, in his article "Banach Limits-Some new thoughts and perspectives" presents a discussion of both classical and recent developments, on the theory of Banach limits. This includes the structure of the set of Banach limits, along with some of its applications to the existence of certain invariant measures, vector valued analogues, functional equations and in the structure theory of Banach spaces. A short description of some recent results of the author on the study of linear selections involving Hahn Banach extensions on spaces of Lipschitz functions on subspaces of Banach spaces, is presented in the last part of the article.

For $n \times n$ matrices with complex entries, the paper by Sukumar on "Some comparative results on eigen values, pseudo spectra and condition spectra" looks at ten variations on the well known eigenvalue theorem with regard to the $\epsilon$-condition spectrum. 


\section{Biography of Professor Kulkarni}

Sudhir Hanmantrao Kulkarni was born in Solapur, Maharashtra in the year 1953. He graduated from S.P. College, affiliated to Pune University, with a B.Sc. degree (1974). He completed his M.Sc. (1976) as well as Ph.D. from IIT Bombay (1980). His thesis title was "Some problems in real Banach algebras". After short stints at the Indian Institute of Science, Bangalore (as a research associate) and at Islamiah Institute of Technology, Bangalore (as an Assistant Professor), he joined the services of IIT Madras in the year 1982. He was promoted as Assistant Professor in July 1988 and as Associate Professor in February 1993. He was appointed as Professor in May 1995. After his retirement in June 2019, he has been associated with IIT Palakkad, Kerala.

Professor Kulkarni is an internationally acclaimed mathematician working in the broad areas of Functional Analysis and Numerical Analysis. He specializes in Banach Algebras, Operator Theory, Generalized Inverses of Linear Operators and their applications to Optimization Theory. His forte is in providing an elementary approach to the solution of many a problem. He has guided ten students in their Ph.D. pursuit and most of them are performing well in academic institutions of national importance. This clearly, is an indication of the quality of education that he has imparted to his students. During his career, he had also collaborated with several expert Functional Analysts in India. He has written a monograph on "Real Function Algebras," published by Marcel Dekker in 1992. He visited the Tuebingen University, Germany as a DAAD fellow during 1994. It will not be an exaggeration to say that Professor Kulkarni has been the face of Functional Analysis at IIT Madras for more than three decades.

Professor Kulkarni has been a teacher par excellence, belonging to the traditional mould and was a silent inspiration to many generations of future mathematicians in the department and elsewhere. He has been associated with Mathematics Teaching and Talent Search Programme of the NBHM for many years. He is a perfect gentleman and always leads by example. His colleagues at all levels have benefited immensely by his wisdom and guidance. He exemplifies the Shakespearean phrase "brevity is the soul of wit".

Here is the list of all the publications by Prof. S. H. Kulkarni.

\section{Publications}

\subsection{Book}

1. Kulkarni, S. H. and Limaye, B. V., Real function algebras. Monographs and Textbooks in Pure and Applied Mathematics, 168. Marcel Dekker, Inc., New York, 1992. viii+186 pp. ISBN: 0-8247-8653-X 


\subsection{Edited volumes}

1. Kulkarni, Rekha P., Kulkarni, S. H., d'Almeida, Filomena D. and Pani, A. K., Editorial note [Topics in functional and numerical analysis]. J. Anal. 14 (2006), iii-iv. 65-06

2. Agrawal Sushama, Kulkarni S. H. and Rao T. S. S. R. K. (Editors) Proceedings of the Eleventh Ramanujan Symposium on Recent Trends in Operator Theory and Banach Algebras held at Ramanujan Institute of Advanced Study in Mathematics Chennai, March 3-5, 2004. Journal of Analysis, Volume 12 (2004).

3. Kulkarni, S. H. and Namboodiri, M. N. N. (Editors) Proceedings of the International Workshop on Linear Algebra, Numerical Functional Analysis and Wavelet Analysis held at Cochin University of Science and Technology, Kochi, August 6-15, 2001. Allied Publishers, Chennai, 2003. ISBN: 81-7764-506-4

\subsection{Research articles}

1. Kulkarni, S. H. and Ramesh, G., Gap formula for symmetric operators, Telangana Academy of Sciences, Volume 01, Year 2020, Pages 129-133.

2. Kulkarni, S. H. and Ramesh, G., Operators that attain reduced minimum, Indian J. Pure Appl. Math., 51(4): December 2020.

3. Kulkarni, S. H. and Ramesh, G., Absolutely minimum attaining closed operators, The Journal of Analysis (2019), https://doi.org/10.1007/s41478-01900189-x

4. Dhara, Kousik, Kulkarni, S. H. and Seidel, M., Continuity of the $(n, \epsilon)$ pseudospectrum in Banach algebras. Integral Equations Operator Theory 91 (2019), no. 4, Paper No. 32, 17 pp.

5. Dhara, Kousik and Kulkarni, S. H., Decomposition of the $(n, \epsilon)$-pseudospectrum of an element of a Banach algebra. Adv. Oper. Theory 5 (2020), no. 1, 248-260.

6. Kulkarni, S. H. and Ramesh, G., On the denseness of minimum attaining operators. Oper. Matrices 12 (2018), no. 3, 699-709

7. Krishnan, Arundhathi and Kulkarni, S. H., Pseudospectra of elements of reduced Banach algebras II. Funct. Anal. Approx. Comput. 10 (2018), no. 2, 33-45.

8. Bhatt, S. J. and Kulkarni, S. H., Gelfand-Mazur theorems in normed algebras: a survey. Expo. Math. 36 (2018), no. 2, 166-177.

9. Dhara, Kousik and Kulkarni, S. H., The $(n, \epsilon)$-pseudospectrum of an element of a Banach algebra. J. Math. Anal. Appl. 464 (2018), no. 1, 939-954

10. Krishnan, Arundhathi and Kulkarni, S. H., Pseudospectra of elements of reduced Banach algebras. Adv. Oper. Theory 2 (2017), no. 4, 475-493.

11. Krishnan, Arundhathi and Kulkarni, S. H., Pseudospectrum of an element of a Banach algebra. Oper. Matrices 11 (2017), no. 1, 263-287. 
12. Kulkarni, S. H., Completeness and invertibility. Math. Student 84 (2015), no. 3-4, 141-145.

13. Kulkarni, S. H., The nullity theorem, its generalization and applications. Semigroups, algebras and operator theory, 149-157, Springer Proc. Math. Stat., 142, Springer, New Delhi, 2015.

14. Kulkarni, S. H., The null space theorem. Linear Algebra Appl. 472 (2015), 97-105

15. Kumar, G. Krishna and Kulkarni, S. H., Banach algebra techniques to compute spectra, pseudospectra and condition spectra of some block operators with continuous symbols. Ann. Funct. Anal. 6 (2015), no. 1, 148-169

16. Kulkarni, S. H., The group of invertible elements of a real Banach algebra. Houston J. Math. 40 (2014), no. 3, 833-836.

17. Krishna Kumar, G. and Kulkarni, S. H., An analogue of the spectral mapping theorem for condition spectrum. Concrete operators, spectral theory, operators in harmonic analysis and approximation, 299-316, Oper. Theory Adv. Appl., 236, Birkhäuser/Springer, Basel, 2014

18. Deepesh, K. P., Kulkarni, S. H. and Nair, M. T., Approximation numbers for relatively bounded operators. Funct. Anal. Approx. Comput. 5 (2013), no. 2, 35-42

19. Deepesh, K. P., Kulkarni, S. H. and Nair, M. T., Generalized inverses and approximation numbers. Combinatorial matrix theory and generalized inverses of matrices, 143-158, Springer, New Delhi, 2013

20. Kumar, G. Krishna and Kulkarni, S. H., Linear maps preserving pseudospectrum and condition spectrum. Banach J. Math. Anal. 6 (2012), no. 1, 45-60.

21. Kulkarni, S. H., Error in "Wiener's theorem, infinite matrices and Banach algebras" [MR2987302]. Math. Newsl. 20 (2011), no. 3, 52.

22. Kulkarni, S. H. and Ramesh, G., Approximation of Moore-Penrose inverse of a closed operator by a sequence of finite rank outer inverses. Funct. Anal. Approx. Comput. 3 (2011), no. 1, 23-32.

23. Kulkarni, S. H. and Ramesh, G., The carrier graph topology. Banach J. Math. Anal. 5 (2011), no. 1, 56-69

24. Kulkarni, S. H., Wiener's theorem, infinite matrices and Banach algebras. Proceedings of the National Symposium on Mathematical Methods and Applications 2009, 23-29, Indian Inst. Technol. Madras, Chennai, 2010.

25. Kulkarni, S. H., Wiener's theorem, infinite matrices and Banach algebras. Math. Newsl. 19 (2010), no. 2, 1-4. 46J10 (46-02)

26. Kulkarni, S. H. and Ramesh, G., Projection methods for computing MoorePenrose inverses of unbounded operators. Indian J. Pure Appl. Math. 41 (2010), no. 5, 647-662

27. Kulkarni, S. H. and Ramesh, G., A formula for gap between two closed operators. Linear Algebra Appl. 432 (2010), no. 11, 3012-3017.

28. Kulkarni, S. H. and Sukumar, D., Almost multiplicative functions on commutative Banach algebras. Studia Math. 197 (2010), no. 1, 93-99.

29. Deepesh, K. P., Kulkarni, S. H. and Nair, M. T., Approximation numbers of operators on normed linear spaces. Integral Equations Operator Theory 65 (2009), no. 4, 529-542. 
30. Kulkarni, S. H., Radha, R. and Sivananthan, S., Non-uniform sampling problem. J. Appl. Funct. Anal. 4 (2009), no. 1, 58-74.

31. Kulkarni, S. H., Nair, M. T. and Ramesh, G., Some properties of unbounded operators with closed range. Proc. Indian Acad. Sci. Math. Sci. 118 (2008), no. 4, 613-625

32. Kulkarni, S. H. and Sukumar, D., The condition spectrum. Acta Sci. Math. (Szeged) 74 (2008), no. 3-4, 625-641.

33. Kulkarni, S. H. and Ramesh, G., Projection methods for inversion of unbounded operators. Indian J. Pure Appl. Math. 39 (2008), no. 2, 185-202.

34. Kulkarni, S. H., A relatively elementary proof of a characterization of polynomials. Indian J. Pure Appl. Math. 38 (2007), no. 4, 229-230.

35. Balasubramanian, R., Kulkarni, S. H. and Radha, R., Solution of a tridiagonal operator equation. Linear Algebra Appl. 414 (2006), no. 1, 389-405.

36. Kulkarni, S. H., Nair, M. T. and Namboodiri, M. N. N., An elementary proof for a characterization of $*$-isomorphisms. Proc. Amer. Math. Soc. 134 (2006), no. 1, 229-234

37. Kulkarni, S. H. and Sukumar, D., Gleason-Kahane-Żelazko theorem for spectrally bounded algebra. Int. J. Math. Math. Sci. 2005, no. 15, 2447-2460.

38. Kulkarni, S. H. and Sivakumar, K. C., Three types of operator monotonicity. J. Anal. 12 (2004), 153-163

39. Agrawal, Sushama and Kulkarni, S. H., Spectral theorem for an unbounded normal operator in a real Hilbert space. J. Anal. 12 (2004), 107-114.

40. Kulkarni, S. H., A very simple and elementary proof of a theorem of Ingelstam. Amer. Math. Monthly 111 (2004), no. 1, 54-58.

41. Balasubramanian, R., Kulkarni, S. H. and Radha, R., Finite dimensional approximations of the operator equations. Linear algebra, numerical functional analysis and wavelet analysis, 57-64, Allied Publ., New Delhi, 2003.

42. Agrawal, Sushama and Kulkarni, S. H., Spectral isometries of real commutative Banach algebras. Report on the Summer Symposium in Real Analysis XXVI. Real Anal. Exchange 2002, 26th Summer Symposium Conference, suppl., 167-172.

43. Agrawal, Sushama N. and Kulkarni, S. H., Spectral representation of a quaternionic Hilbert space. Math. Today (Ahmedabad) 19 (2001), no. 1, 3-8.

44. Balasubramanian, R., Kulkarni, S. H. and Radha, R., Non-invertibility of certain almost Mathieu operators. Proc. Amer. Math. Soc. 129 (2001), no. 7, 2017-2018.

45. Agrawal, Sushama and Kulkarni, S. H., An analogue of the Riesz-representation theorem. Novi Sad J. Math. 30 (2000), no. 1, 143-154.

46. Agrawal, Sushama and Kulkarni, S. H., Dual spaces of quaternion normed linear spaces and reflexivity. J. Anal. 8 (2000), 79-90.

47. Kulkarni, S. H., Arzela-Ascoli theorem is stable. Internat. J. Math. Ed. Sci. Tech. 31 (2000), no. 6, 919-922.

48. Kulkarni, S. H. and Nair, M. T., A characterization of closed range operators. Indian J. Pure Appl. Math. 31 (2000), no. 4, 353-361. 
49. Kulkarni, S. H. and Limaye, B. V., Boundaries and Choquet sets for real subspaces of C(X). Math. Japon. 51 (2000), no. 2, 199-212

50. Agrawal, Sushama and Kulkarni, S. H., Some applications of a special theorem for a normal operator on a real Hilbert space. Math. Today (Ahmedabad) 16 (1998), 65-76

51. Bhatt, S. J., Karia, D. J., Kulkarni, S. H. and Shimpi, M. E., A note on the Gelfand-Mazur theorem. Proc. Amer. Math. Soc. 126 (1998), no. 10, 2999-3005.

52. Kulkarni, S. H. and Sivakumar, K. C., Explicit solutions of a special class of linear programming problems in Banach spaces. Acta Sci. Math. (Szeged) 62 (1996), no. 3-4, 457-465

53. Kulkarni, S. H. and Sivakumar, K. C., Applications of generalized inverses to interval linear programs in Hilbert spaces. Numer. Funct. Anal. Optim. 16 (1995), no. 7-8, 965-973.

54. Kulkarni, S. H. and Sivakumar, K. C., Explicit solvability of a class of infinite linear economic models. Indian J. Pure Appl. Math. 26 (1995), no. 3, 217-223.

55. Kulkarni, S. H. and Limaye, B. V., Extreme points of the unit ball in the dual spaces of some real subspaces of C(X). Glas. Mat. Ser. III 29(49) (1994), no. 2, 333-340.

56. Agrawal, Sushama N. and Kulkarni, S. H., A spectral theorem for a normal operator on a real Hilbert space. Acta Sci. Math. (Szeged) 59 (1994), no. 3-4, $441-451$

57. Agrawal, Sushama and Kulkarni, S. H., Intrinsic proof for real analogue of Fuglede Putnam Rosenblum theorem. Math. Today 12 (1994), 3-6. 46L05

58. Kulkarni, S. H., Representation of a real $B^{*}$-algebra on a quaternionic Hilbert space. Proc. Amer. Math. Soc. 121 (1994), no. 2, 505-509.

59. Kulkarni, S. H. and Arundhathi, S., A note on the metric topology on Gleason parts of a real function algebra. Bull. Calcutta Math. Soc. 85 (1993), no. 5, 413-418

60. Kulkarni, S. H., Representations of a class of real $B^{*}$-algebras as algebras of quaternion-valued functions. Proc. Amer. Math. Soc. 116 (1992), no. 1, 61-66.

61. Kulkarni, S. H. and Arundhathi, S., Isometries of real function algebras. Comment. Math. Prace Mat. 30 (1991), no. 2, 343-356.

62. Kulkarni, S. H. and Srinivasan, N., An analogue of Wermer's theorem for a real function algebra. Math. Today 8 (1990), 33-42

63. Kulkarni, S. H. and Srinivasan, N., Erratum: "An analogue of HoffmanWermer theorem for a real function algebra". Indian J. Pure Appl. Math. 20 (1989), no. 1, 98

64. Kulkarni, S. H., Topological conditions for commutativity of a real Banach algebra. Houston J. Math. 14 (1988), no. 2, 235-245

65. Kulkarni, S. H. and Srinivasan, N., A note on a theorem of Gorin. Math. Japon. 33 (1988), no. 6, 887-893

66. Kulkarni, S. H. and Arundhathi, S., Choquet boundary for real function algebras. Canad. J. Math. 40 (1988), no. 5, 1084-1104.

67. Srinivasan, N. and Kulkarni, S. H., Restriction algebras of a real function algebra. J. Math. Phys. Sci. 22 (1988), no. 2, 209-223 
68. Kulkarni, S. H. and Srinivasan, N., An analogue of Hoffman-Wermer theorem for a real function algebra. Indian J. Pure Appl. Math. 19 (1988), no. 2, 154-166.

69. Kulkarni, S. H. and Srinivasan, N., An analogue of Bishop's theorem for real function algebras. Indian J. Pure Appl. Math. 18 (1987), no. 2, 136-145.

70. Kulkarni, S. H. and Limaye, B. V., Isometry of an analytic map onto a Gleason part. Glas. Mat. Ser. III 21(41) (1986), no. 2, 353-356.

71. Arundhathi, S. and Kulkarni, S. H., Analytic and harmonic maps into a topological space. Proc. Indian Acad. Sci. Math. Sci. 95 (1986), no. 1, 37-40

72. Kulkarni, S. H., Gleason-Kahane-Żelazko theorem for real Banach algebras. J. Math. Phys. Sci. 18 (1983/84), Special Issue, S19-S28.

73. Kulkarni, S. H., Fourier transforms of physical functions. II. Infinitely many oscillations. Indian J. Pure Appl. Math. 14 (1983), no. 9, 1183-1193.

74. Kulkarni, S. H., Fourier transforms of physical functions. I. Infinite differentiability. Indian J. Pure Appl. Math. 14 (1983), no. 8, 980-993.

75. Kulkarni, S. H., Analytic and harmonic maps into the maximal ideal space of a function algebra. J. Math. Phys. Sci. 17 (1983), no. 2, 169-175.

76. Kulkarni, S. H. and Limaye, B. V., A topological characterization of Gleason parts of real function algebras. Canad. Math. Bull. 26 (1983), no. 1, 44-49.

77. Kulkarni, S. H. and Limaye, B. V., Spectral mapping theorem for real Banach algebras. Houston J. Math. 7 (1981), no. 4, 507-517

78. Kulkarni, S. H. and Limaye, B. V., Gleason parts of real function algebras. Canadian J. Math. 33 (1981), no. 1, 181-200

79. Kulkarni, S. H., A note on the Shirali-Ford theorem. Math. Student 48 (1980), no. 2-4, 343-347 (1984)

80. Kulkarni, S. H. and Limaye, B. V., Gelfand-Naimark theorems for real Banach *-algebras. Math. Japon. 25 (1980), no. 5, 545-558.

Publisher's Note Springer Nature remains neutral with regard to jurisdictional claims in published maps and institutional affiliations. 